\title{
Benjamin Libet und der freie Wille
}

\author{
Robert Reimer \\ Universität Leipzig \\ Institut für Philosophie
}

\section{Zusammenfassung}

Am Ende einer Reihe von wegweisenden Experimenten resümierte der Kognitionswissenschaftler Benjamin Libet enttäuscht, dass der freie Wille eine Illusion sein müsse. Aufgefordert eine bestimmte Körperbewegung zu vollziehen, konnten in den Gehirnen seiner Proband*innen schon kurz vor dem Bewusstwerden der Handlungsabsicht neuronale Aktivitäten nachgewiesen werden konnten, die typisch für die entsprechende Bewegung sind. Doch das implizite Verständnis der Natur des freien Willens, das diesen Versuchen zugrunde liegt, ist problematisch und hat seine Wurzeln in der philosophischen Idee des interaktionistischen Substanzdualismus von René Descartes. In diesem Aufsatz werde ich dieses Verständnis offenlegen und historisch verorten. Zum Schluss werde ich noch eine plausiblere Alternative anbieten.

\section{René Descartes und die res cogitans}

Bis zum Aufkommen der Kognitionswissenschaften war die Frage, ob wir einen freien Willen haben, vor allem eine philosophische Frage. Der freie Wille war nicht Gegenstand empirischer Forschung. Und in der Tat scheint die Idee, man könne den freien Willen experimentell nachweisen, zunächst absurd. Worin könnte ein solcher Nachweis bestehen? Bezeichnet der Ausdruck >freier Wille die Funktionsweise eines Organs? Hat eine Person einen freien Willen so wie sie einen Stoffwechsel hat? Und wenn ja, wie wirkt er, und vor allem, wo?

Die Vorstellung, der freie Wille müsse sich irgendwo im menschlichen Körper manifestieren, ist in der Tat schon älter als die Kognitionswissenschaften und soll eine Antwort auf die Frage liefern, wie der Mensch selbstbestimmt in die Welt eingreifen kann. Das Problem: Wie Steine, Tiere und Pflanzen sind auch wir Teil verschiedener Kausalketten, die unser Tun bestimmen. Essen wir etwas, verdauen wir es und scheiden es wieder aus. Schlägt uns jemand mit einem Hammer gegen das Knie, treten wir zurück, dank des Kniesehnenreflexes. Es scheint, als seien für viele unserer Bewegungen problemlos äußere Ursachen anzugeben. Auch wenn wir aktuell

Erschienen in: arbeitstitel - Forum für Leipziger Promovierende, Band 7, Heft 1 (2019), S. 17-20. Lizenzierung CC-BY-SA 4.0. nicht alle dieser Kausalketten aufdecken können, gibt es keinen Grund anzunehmen, wir könnten auch nur manchmal frei von jeglichen äußeren Bestimmungen handeln.

Dem Philosophen René Descartes bereitete diese Vorstellung, die wir noch heute mit dem Titel >Determinismus` versehen, große Sorgen. Beeindruckt von den Erklärungskraft der damals aufkeimenden kepler'schen und galilei'schen Mechanik, sah er die Idee des freien Willens bedroht und versuchte sie mit der Idee, alle Geschehnisse seien Teil kausaler Ketten, in Einklang zu bringen. Ihm zufolge gibt es zwei Arten von Substanzen: die Dinge (res extsensa), deren Bewegungen durch kausale Wirkmechanismen bestimmt sind, und die Geist- oder Seelensubstanz (res cogitans), deren Operationen nicht kausal determiniert und worin der freie Wille sich entfaltet. Nun existieren beide Substanzen nicht nebeneinanderher, sondern sind eng miteinander verflochten. Doch Descartes Ausführungen darüber, wie diese Verflechtung zu verstehen ist, sind nicht eindeutig. An einigen Stellen vertritt Descartes eine Aristotelische Auffassung, der zufolge die Seele die Form des gesamten Körpers ist und ihren Sitz im ,[...] gesamten Verband seiner Organe [...]" (Descartes, 2014, § 30) hat. Gleichzeitig weiß Descartes, der sich intensiv mit der Anatomie und Biologie des menschlichen Körpers auseinandergesetzt hat, um die zentrale Rolle des Gehirns für die Ausführung willentlicher Handlungen. Entsprechend vermutet er auch, dass es einen primären Manifestationsort der Funktionen der res cogitans geben muss - nämlich die Zirbeldrüse. So schreibt er in >Die Passionen der Seele<:

„Wir müssen es also so auffassen, dass die Seele ihren Hauptsitz in der kleinen, sich in der Mitte des Gehirns befindenden Drüse hat. Von dort strahlt sie durch Vermittlung des Spiritus, der Nerven und sogar des Blutes [...] auf den gesamten Rest des Körpers aus [...]. Das führt dazu, dass die in seinen Hohlräumen enthaltenen Lebensgeister verschieden in die Muskeln eintreten, wodurch sie die Körperglieder auf alle verschiedenen Weisen bewegen können, zu denen sie imstande sind." (Descartes, 2014, § 34)

Descartes ${ }^{6}$ Denkweise firmiert in der Philosophie des Geistes unter dem Titel >interaktionistischer Substanzdualismus $<$ und wird heute kaum noch explizit vertreten, da zwar die Zirbeldrüse im Gehirn, aber nicht das Zusammenspiel zwischen res extensa, Zirbeldrüse und Gehirn nachgewiesen werden konnte. Dennoch vermute ich, dass die allgemeine Vorstellung einer Einflussnahme des Geistes auf den Körper die Auffassung von einem freien 
Willen nachhaltig beeinflusst und sich auch in die Kognitionswissenschaften eingeschlichen hat. Nach Descartes vollzieht die res cogitans eine Art >Willensakt<, und greift so in den Lauf der res extensa ein. Sie >steuert den Körper als wäre er eine Maschine, ist aber in ihrem Wirken umgekehrt nicht durch deren Einflüsse determiniert. Zwar empfängt die res cogitans durch die Zirbeldrüse Wahrnehmungsinformationen. Was sie mit diesen anstellt, ist jedoch ihr überlassen. Für den Willen bedeutet dies, dass er , ,...] von seiner Natur her solchermaßen frei [ist], dass er niemals gezwungen werden kann." (Descartes, 2014, § 41) Die kausale Kette wird unterbrochen, sobald etwas die res cogitans >betritt< und beginnt von Neuem, sobald der freie Wille wirksam wird. Die res cogitans, die die Willensakte vollzieht, ist also eine Art >unbewegten Beweger .

\section{Benjamin Libet und seine Experimente}

Sie werden sich nun sicher fragen, was Descartes mit dem Kognitionswissenschaftler Benjamin Libet zu tun hat? Libet ging nie davon aus, dass der freie Wille in einer übersinnlichen Substanz beheimatet ist. Dennoch gibt es eine Gemeinsamkeit: Descartes nahm an, dass der freie Wille als kausale Wirksamkeit verstanden werden muss, die jedoch selbst nicht kausal determiniert ist. Libet, der eigentlich die Existenz des freien Willens beweisen wollte, am Ende seiner Experimente diese allerdings in $\mathrm{Ge}$ fahr sah, vertrat dieselbe Annahme. Und genau in dieser Annahme liegt das fundamentale Fehlverständnis, das seinen Experimenten vorausgeht.

Aber zunächst ein paar Worte zum Aufbau der LibetExperimente. Ich werde Ihnen eine Kurzzusammenfassung ohne genaue Messergebnisse und ohne die Feinheiten der statistischen Auswertung geben. Ob Libet wissenschaftlich korrekt gearbeitet und ausgewertet hat, spielt für meine Argumentation aber auch keine Rolle. Mich interessieren ausschließlich die impliziten Annahmen, über die Natur dessen, was er in seinen Experimenten nachweisen wollte.

Libets Forschungsziel bestand darin, zu klären, wann und in welchem Zusammenhang der bewusste Wunsch oder die Absicht für eine bestimmte Handlung >auftritt<: ,[W]hen does the conscious wish or intention (to perform an act) appear?"“ (Libet, 1999, p. 49). Dafür sollten Proband*innen zu einem beliebigen Zeitpunkt innerhalb eines Intervalls von 30 Sekunden die Hand bewegen und mit Hilfe einer Uhr möglichst genau bestimmen, wann sie den Wunsch (>wish $<$ ), die Absicht (>intention<), bzw. den Drang ( >urge $<$ ) zu dieser Bewegung verspürten. Libet bezeichnete diesen >conscious will to act (Libet, 1999, p. 50) auch als Willensakt und kürzte ihn mit $>\mathrm{W}$ < ab, sowie die Bewegung selbst mit `EMG . Nun stellte er fest, dass bereits einige Millisekunden bevor die Proband*innen (W) verspürten, eine unbewusste Aktivität im Motorkortex gemessen werden konnte - das sogenannte >Bereitschaftspotenzial $<$, oder $>$ Readiness Potenzial $<(>\mathrm{RP}<)-$ das für die entsprechende Bewegung typisch ist. Libet vermutete daher, dass der Willensakt nur das Ergebnis dieser unbewussten Prozesse sein könnte:

,$[\mathrm{R}]$ elatively >spontaneous $<$ voluntary acts, performed without conscious deliberation or planning, may also be initiated by cerebral activities proceeding unconsciously. These considerations would appear to introduce certain constraints on the potential of the individual for exerting conscious initiation and control over his voluntary acts." (Libet, 1983, p. 641)

Die Idee, dass die Handlungen durch unbewusste Hirnvorgänge und nicht durch die freie Willkür der handelnden Person bestimmt sind, widerspricht Descartes ' interaktionistischem Substanzdualismus. Egal, ob der von den Proband*innen empfundene Willensakt (W) ein bloßes Epiphänomen ist oder durchaus real und kausal wirksam, seine Prädetermination durch andere unbewusste Prozesse war für Libet Anlass genug zu glauben, dass die Freiheit des Willens eine Illusion sein muss. Descartes und Libet kommen also zu einem anderen Schluss, teilen aber die gleiche metaphysische Annahme, dass der freie Wille ein Akt sein müsse, der selbst frei von körperlichen Einflüssen, den Körper zum Handeln bringt.

Um die Wirkungsweise dieses Willensaktes zu umschreiben, bedienen sich sowohl Descartes als auch Libet verschiedener Wendungen, die die Interaktion zweier Gegenstände artikulieren. Während Descartes häufig transitive Verben der Hervorbringung wie >faire que ( $>$ machen, dass $<$ ) oder >produire $<$ ( $>$ produzieren $\measuredangle$ ) in $\S 41$ verwendet, und auch Verben für mechanische Prozesse wie >agir sur qc. ( >auf etwas einwirken ) oder >mouvoir qc. (>etwas bewegen $\iota$ ) in $\S 34$; wählt Libet eher neutrale Verben der kausalen Einflussnahme wie >initiate sth.<, >affect sth.<, >control sth.< oder >influence sth.<, so zum Beispiel in: "I myself proposed an experimental design that could test whether conscious will could influence nerve cell activities in the brain [...]" (Libet, 1999, p. 56). Alle drei Verbtypen stellen den Willensakt als etwas dar, das autonom in neuronale oder biologische Prozesse gewissermaßen von außen oder sogar >aus dem Nichts` eingreifen kann. Nach Libet müssten freie Willensakte, wenn sie denn existieren, also genau so funktionieren wie neuronale Prozesse, nämlich die Nervenzellen triggern, damit diese die entsprechende Muskelbewegung (EMG) hervorbringen. Gleichzeitig dürfen sie nicht selbst durch einen neuronalen Prozess wie das (RP) getriggert werden, dürfen also kein bloßes Glied neben anderen Gliedern in der Kette neuronaler Prozesse sein.

\section{Das Oppositionsverhältnis zwischen Körper und Geist}

Ich vermute, dass sowohl Descartes als auch Libet eine problematische Vorstellung über die Natur und Wirkungsweise des freien Willens haben - eine Vorstellung, die im Widerspruch sowohl zu unserer eigenen alltäglichen Verwendung des Begriffs als auch zum Großteil der Philosophiegeschichte steht. Der Geist (bei Descartes), bzw. das Bewusstsein (bei Libet) wird hier als ein Pilot vorgestellt, der frei sein Flugzeug über die entsprechenden Schaltknüppel (den Körper über das Gehirn) steuert. Viele Folgeexperimente, die zeigen wollten, dass der freie doch Wille existiert, versuchten andere geplante oder spontane Willensakte, bzw. Handlungsunterlassun- 
gen (>im letzten Moment $\varsigma$ ) experimentell aufzuspüren, denen keine bestimmte neuronale Aktivität (RP) nachgewiesen werden konnte. Ich fürchte aber, dass dieses Unterfangen im Allgemeinen wenig vielversprechend ist. Stattdessen sollten wir die Vorstellung fallenlassen, die Freiheit des Willens würde sich in einem indeterminierten Eingriff des Geistes in die Prozesse des Körpers äußern, und damit auch die Vorstellung, im Handeln befänden sich Geist und Körper in einem Oppositionsverhältnis einer einseitigen kausalen Einflussnahme zueinander. In der Tat gibt es viele Wendungen, die ausdrücken, dass wir in Opposition zu unserem eigenen Körper stehen und einige davon verwendet Libet selbst: Wir können uns selbst kontrollieren, bzw. unseren Körper nicht unter Kontrolle haben oder einen Drang (>urge $\iota)$ unterdrücken (〉suppress $\triangleleft$ ). Aber all diese Phänomene beschreiben nicht, was geschieht, wenn wir eine einfache freie Körperbewegung ausführen. Der Begriffe der Kontrolle bspw. impliziert ein Verhältnis zwischen Akteur*innen und eigenständigen von ihnen zunächst unabhängigen Prozessen, die in ihrem Lauf umgelenkt werden müssen. Unser Flugzeug bspw. kann kontrolliert werden, ebenso wie ein Niesreflex. Der Reflex würde ohne bewusstes $\mathrm{Zu}-$ tun einfach ablaufen und das Flugzeug (bis zum drohenden Absturz) weiterfliegen. Um eigene körperliche oder fremde mechanische Prozesse $\mathrm{zu}$ kontrollieren, müssen wir zunächst erspüren (im Fall des Niesens) oder beobachten (im Falle des Flugzeugs), dass und wie sie ablaufen oder herannahen. Wir nehmen also zunächst eine passive rezeptive oder propriozeptive Haltung gegenüber ihnen ein. Dann müssen wir aktiv eine Gegenhandlung ausführen. Um das Flugzeug unter unsere Kontrolle zu bringen, ergreifen wir den Schaltknüppel und verändern so die Flugrichtung. Um unseren Niesanfall unter Kontrolle zu bringen und seine Wirkung einzudämmen, können wir die Lippen oder den Rachenraum zusammenpressen.

All das trifft für Körperbewegungen nicht zu. Es ist nicht so, dass wir oder unser Geist kontrollierenden Einfluss auf unsere neuronalen Prozesse, bzw. unsere Muskeln nehmen müssen, um so eine freie Körperbewegung auszuführen. Im Handeln ist unser Körper keine uns entgegenstehende >Maschine , die einfach läuft und die wir (oder unser Geist) zügeln und in die rechten Bahnen lenken müssen. Alle im letzten Paragraphen beschriebenen Gegenhandlungen oder kontrollierende Einflussnahmen (das Greifen nach dem Schaltknüppel oder das Zusammenpressen der Lippen) sind selbst einfache Handlungen, bzw. Körperbewegungen, die nicht wiederum kontrolliert werden müssen, um korrekt ausgeführt werden zu können. Salopp gesprochen: Wir tun sie einfach, ohne dabei etwas zu kontrollieren. Das interaktionistische Erklärungsmodell von Descartes und Libet schiebt einen Keil zwischen den oder die Handelnde, bzw. den Geist und den Körper in der Ausführung grundlegender Körperbewegungen, der keinerlei phänomenologische Grundlage hat. Im freien Handeln sind wir vielmehr eins mit unserem Körper und insbesondere mit den Körperteilen, die wir bewegen, und es scheint keinerlei vermittelnde Instanzen oder Prozesse zu geben. Daraus folgt, dass der von Libet's Proband*innen dokumentierte Drang zu handeln, bzw. die Absicht oder der Wunsch kein Willensakt in dem von mir eben beschriebenen interaktionistischen Sinne sein kann. Was aber ist dann dasjenige, das wir benennen, wenn wir drauf und dran sind zu handeln?

So wie die Philosophin Elizabeth Anscombe in ihrem Buch >Intention` (Anscombe, 1957) lehne auch ich die Vorstellung ab, das Wort >Absicht « bezeichne ein zeitlich vorhergehendes mentales Ereignis, das die Handlung kausal hervorbringt, bzw. triggert. Und wie sie (und manchmal auch Descartes) neige auch ich zu der durch Aristoteles geprägten hylemorphistischen Vorstellung, dass die Begriffe >Seele< oder >Geist< eher die Form des Menschen bezeichnen, also die Art und Weise, wie Menschen(körper) geformt sind und agieren. Demnach sind der Geist oder die Seele nicht mehr ein >Ding anderen >Ding und dementsprechend sind auch Absichten keine Trigger, die von dem einen Ding aus das andere anstoßen. Vielmehr handelt es sich bei der Absicht um einen Zustand, in dem sich die handelnde Person befindet, während sie zielgerichtet handelt, und der den Verlauf der Handlung bestimmt. So könnte auch die verdinglichende Formulierung >eine Absicht zu haben besser durch die Verbalphrase >mit Blick auf ein bestimmtes Ziel handelnd ‘ ausgedrückt werden.

Klarerweise gehen auch mit dieser Auffassung einige schwierige philosophische Fragen einher, insbesondere die, in welchem Verhältnis denn dann dieser >AbsichtsZustand $\mathrm{zu}$ den neuronalen Prozessen im Gehirn steht, die ja (erwiesenermaßen) immer noch außerordentlich wichtig für das Gelingen der Handlung sind? Eine ausführliche Antwort auf diese Frage kann ich an dieser Stelle nicht geben. Nur so viel: So, wie Absichten, haben alle geistigen oder mentalen Phänomene einer Person (Schmerzen, Gedanken, Erinnerungen) neuronale Korrelate. Mit dem Ausdruck >neuronales Korrelat bezeichnen wir in der Philosophie die materielle Grundlage für mentale Phänomene - und zwar bestimmte Prozesse im Nervensystem. Die neuronalen Korrelate verhalten sich $\mathrm{zu}$ den entsprechenden mentalen Phänomenen (Absichten) nicht wie Ursache und Wirkung. Daher macht es auch keinen Sinn nach einer Schnittstelle im Gehirn zu suchen, wo ein neuronaler Prozess von einem mentalen Phänomen (oder umgekehrt) getriggert wird. Ein mentales Phänomen und sein dazugehöriges neuronales Korrelat verhalten sich zueinander eher wie zwei Seiten ein und derselben Medaille. Während wir mit einer bestimmten Absicht handeln, ereignen sich in unserem Körper und Nervensystem Prozesse, die die nötige materielle Basis für diese Handlung bilden, indem sie die entsprechende Muskelbewegung antreiben, die die Handlung ermöglichen. Die Annahme, man müsse, um frei Handeln zu können, in den Verlauf dieser unbewussten Prozesse eingreifen können, ähnelt dem verzweifelten und aussichtslosen Wunsch im Laufen seinen eigenen Schatten einzuholen.

\section{Die Frage nach dem freien Willen}

Nach dieser Kritik müssen wir jedoch noch immer die schwierige und entscheidende Frage beantworten, ob der Determinismus ein Problem für den freien Willen darstellt, denn die Annahme, dass für jede bewusste Handlung ein zeitlich vorhergehendes (RP) gemessen werden 
kann, ist noch nicht vom Tisch. Auch wenn es stimmt, dass Körper und Geist im Handeln nicht in einem Oppositionsverhältnis stehen, bleibt die empirisch nachweisbare Tatsache bestehen, dass neuronale Vorgänge gemessen werden können, die für die Ausführung einer Handlung hinreichend und notwendiger sind, und zwar schon bevor der Handelnden selbst die Ausführung dieser Handlung bewusst wird. Macht sie das nicht dennoch unfrei?

Um diese Frage zu beantworten, werde ich einen alternativen Verständnisvorschlag für die Natur des freien Willens anbieten, der mit dem eben beschriebenen Determinismus kompatibel ist. Statt einen Akt, bezeichnet der Ausdruck >freier Wille` ein Vermögen, nämlich das Vermögen Gründe für eine mögliche Handlung abzuwägen, Pläne zu machen, im Handeln Handlungsziele zu repräsentieren und auch zu ändern, sich seiner eigenen Handlungsgewohnheiten bewusst $\mathrm{zu}$ werden, und das eigene Handeln kritisch zu hinterfragen. Um dieses Vermögen $\mathrm{zu}$ besitzen sind wir sogar darauf angewiesen, über ein funktionierendes Nervensystem zu verfügen. Die Tatsache, dass bewusste Handlungen auf Basis der Messung der entsprechenden neuronalen Prozesse vorhergesehen werden kann, hat prima facie keinen Einfluss auf den Besitz dieses Vermögens. Ich nehme Ihnen die Freiheit, wenn ich dafür sorge, dass Sie die von Ihnen gefasste Absicht nicht durchsetzen können (bspw. indem ich Sie fessele oder sie mit Rauschmitteln unzurechnungsfähig ma- che). Ich nehme Ihnen die Freiheit nicht, wenn ich bloß voraussagen kann, was Sie tun werden. Der bloße Umstand der Voraussagbarkeit ist noch keine Gefahr für den freien Willen. Er wird erst dann zu einer Gefahr, wenn tatsächlich eine Person Ihre Handlung auf Basis der Auswertung Ihrer neuronalen Aktivitäten prognostiziert und dann mit Hilfe dieser Prognose aktiv ihr Vorhaben zu vereiteln oder $\mathrm{zu}$ unterbinden versucht. So eine Art der $>$ Freiheitsberaubung « wird bspw. in dem Film >Minority Report $<$ thematisiert.

\section{Literatur}

Anscombe, G. E. (1957) Intention. Cambridge: Harvard University Press.

Descartes, R. (2014) Die Passionen der Seele. (Originaltitel: Les Passions de l'âme.) Aus dem Französischen und hg. von Christian Wohlers. Hamburg: Felix Meiner Verlag.

Libet, B. (1999) Do We Have a Free Will? Journal of Consciousness Studies, (6 (8-9), S. 47-57.

Libet, et al. (1983) Libet, B., Gleason, C. A., Wright, E. W., \& Pearl, D. K.-p. Time of conscious intention to act in relation to onset of cerebral activity (readinesspotential). The unconscious initiation of a freely voluntary act. Brain: a Journal of Neurology, (106 (pt 3)), S. 623-642. 